\title{
ASSOCIATIVE $H$-SPACES WITH SMALL RANKS
}

\section{J. R. HUBBUCK}

Abstract. Let $X$ be a finite connected CW-complex which is a topological monoid. It is shown that if the rank of $X$ is less than five, then the rational type of $X$ is that of a Lie group.

0. The classification of the homotopy types of complexes with few cells which support $H$-space multiplications now seems significantly nearer completion than was the case just a short time ago, [8] and [17]. If one can show that the only possible rational cohomology rings of these $H$-spaces are those which are known to occur, then one might hope to generate all possible homotopy types from the known examples using Zabrodsky's technique of mixing rationally equivalent $H$-spaces [16]. Now to determine the rational cohomology rings of $H$-spaces with few cells is not an easy problem. In fact without information on torsion in the integral homology and/or some geometric or Hopf algebraic associativity assumption connected with the $H$ space multiplication, very little is known. In this note we consider the rational cohomology rings of such $H$-spaces where we make no torsion assumptions but where the $H$-space multiplication is strictly associative.

1. Let $X$ be a finite connected CW-complex which supports an $H$-space multiplication. The rational cohomology ring $H^{*}(X, Q)$, is isomorphic to that of a product of odd dimensional spheres [9]. The number of spheres, $r(X)$, is called the rank of $X$. We define $t(X)$, the type of $X$, to be the set of integers $\{q\}$ where $\{2 q-1\}$ is the set of dimensions of these spheres. This is not quite the usual definition of type but it is convenient here.

THeOREM 1.1. Let $X$ be a finite connected CW-complex which supports a strictly associative $H$-space multiplication. If $r(X) \leqq 4$; then $t(X)=t(G)$ where $G$ is a Lie group.

Representatives for all the Lie groups which occur in Theorem 1.1 can be obtained from products of $S^{1} ; S U(n), 2 \leqq n \leqq 5 ; S p(n), 2 \leqq n \leqq 4$; Spin (8); $G_{2}$ and $F_{4}$, of the appropriate ranks. This result for $r(X)=2$

Received by the editors May 4, 1970.

AMS 1969 subject classifications. Primary 5540.

$K e y$ words and phrases. Connected finite $\mathrm{CW}$-complex, associative $H$-space, classifying space, Steenrod algebra. 
is due to L. Smith [14] and a partial result for $r(X)=3$ is contained in $[12]$ and $[13]$.

There is one main technical result used in the proof of Theorem 1.1 and this is our next theorem.

THEOREM 1.2. Let $X$ be a connected finite complex which is an associative $H$-space. Suppose that $r-1$ and $m$ are relatively prime integers. If $m \in t(X)$, then $q \in t(X)$ for some $q$ with $q=r \bmod m$.

Let $\phi(n)$ be the Euler function of $n$-the number of integers not greater than $n$ which are prime to $n$. An immediate consequence of Theorem 1.2 is a result of Ochiai [13].

Corollary 1.3. If $m \in t(X)$, then $r(X) \geqq \phi(m)$.

The simple technique of this paper was first used by A. Clark in [5]. Sometime later the author discovered Theorem 1.2, which is essentially contained in [5], as a corollary to a more general theorem proved using complex $K$-theory, but it is a modified version of Clark's argument which we use here. We take the classification of Theorem 1.1 to the point where these simple arguments appear to fail to give a complete solution. Thus to eliminate one possible type of rank 4 we use the generalized reduced powers of [10] and [11]. (In fact, one reason for defining these homomorphisms in the first place was to relate the two different proofs of Theorem 1.2.) The increased interest in finite dimensional $H$-spaces suggests that it is now worthwhile writing down Theorem 1.2 quite explicitly. The proof is given in $\$ 2$ and Theorem 1.1 is proved in $\S 3$, apart from the one exception mentioned above. This special case is considered in $\$ 4$.

2. Let $X$ be a finite connected complex which is an associative $H$-space. Let $P$ be the set of primes for which the integral homology of $X$ has no torsion. As $X$ is a finite complex this is all but a finite number of primes. An associative $H$-space has a classifying space [7], and for $p \in P, H^{*}\left(B X, Z_{p}\right)$ is a polynomial algebra on generators with dimensions $\{2 q\}$, where $\{q\}=t(X)[1]$. Also $H^{*}(B X, Q)$ is a polynomial algebra on generators with these same dimensions.

We consider the action of the Steenrod algebra $a(p)$ on $H^{*}\left(B X, Z_{p}\right)$ for $p \in P$. The Cartan formula for the cyclic reduced powers

$$
P^{q}(x y)=\sum_{i+j=q} P^{i}(x) \cdot P^{j}(y)
$$

ensures that the truncated polynomial algebra $A^{p}$ which we obtain as a quotient ring of $H^{*}\left(B X, Z_{p}\right)$ by truncating at height $p+1$, is also an unstable $a(p)$-module. Let $Q\left(A^{p}\right)$ be the indecomposable 
quotient module of $A^{p}$. If $p=2, P^{i}$ now stands for the Steenrod square $\mathrm{Sq}^{2 i}$.

Lemma 2.1. Suppose that $P^{m}\left(A_{2 m}^{p}\right) \subset P^{1}\left(A_{2 p m-2(p-1)}^{p}\right)$.

(a) If $Q\left(A^{p}\right)_{2 m} \neq 0$, then $Q\left(A^{p}\right)_{2 q} \neq 0$ for some $q$ with $q+(p-1)=0$ $\bmod m$.

(b) If the two conditions $Q\left(A^{p}\right)_{2 q} \neq 0$ and $q+(p-1)=0 \bmod m$ imply that $q=m-(p-1)$, then $P^{1}: Q\left(A^{p}\right)_{2 q} \rightarrow Q\left(A^{p}\right)_{2 m}$ is surjective.

(c) If the two conditions $Q\left(A^{p}\right)_{2 q} \neq 0$ and $q+(p-1)=0 \bmod m$ have a unique solution for $q$ and also $P^{1}: Q\left(A^{p}\right)_{2 q} \rightarrow Q\left(A^{p}\right)_{2 q+2(p-1)}$ is injective, then either $q=m-(p-1)$ or $Q\left(A^{p}\right)_{2 m}=0$.

The condition that $P^{m}\left(A_{2 m}^{p}\right) \subset P^{1}\left(A_{2 p m-2(p-1)}^{p}\right)$ is always satisfied if $m$ is not divisible by $p$ because of the Adem relation

$$
P^{1} P^{m-1}=m P^{m} \text {. }
$$

The proof of Lemma 2.1 is based on the following observations. Let $x_{1}, x_{2}, \cdots, x_{r}$ be a homogeneous multiplicative basis for $A^{p}$ and suppose that $P^{1}\left(x_{1}^{l} x_{2}^{m} \cdots\right)=\sum a(\alpha, \beta, \cdots) x_{1}^{\alpha} x_{2}^{\beta} \cdots$, a linear combination of distinct monomials in the $x_{i}$. If $a(\alpha, 0,0, \cdots, 0) \neq 0$ where $\alpha \leqq p$, then $x_{1}^{l} x_{2}^{m} \ldots=x_{1}^{l}$ or $x_{1}^{l} x_{s}, 2 \leqq s \leqq r$, for otherwise the Cartan formula for $P^{1}$ implies that each summand on the right-hand side involves the product of at least two distinct generators. Further $P^{1} x_{s}=\sum b(\alpha, \beta, \cdots) x_{1}^{\alpha} x_{2}^{\beta} \cdots$, where $b(\alpha, 0,0, \cdots, 0) \neq 0$ for some $s$ in $1 \leqq s \leqq r$. Similarly if $P^{1} w=x_{1}^{p}$ for some $w$ and we express $w$ as a sum of monomials in the $x_{i}$, the linearity of $P^{1}$ implies that at least one of these monomials satisfies the first condition above and so there exists a generator $x$, with

$$
P^{1} x_{s}=\sum b(\alpha, \beta, \cdots) x_{1} \stackrel{\alpha}{x}_{2}^{\beta} \cdots, \quad \text { where } b(\alpha, 0,0, \cdots, 0) \neq 0 .
$$

The proof of Lemma 2.1 (a). We may choose $x_{1} \in A_{2 m}^{p}$ which must have nonzero image in $Q\left(A^{p}\right)$. Since $x_{1} \in A_{2 m}^{p}$,

$$
P^{m} x_{1}=x_{1}^{p}
$$

which is nonzero in $A^{p}$, and so by hypothesis there exists an element $w$ such that $P^{1} w=x_{1}^{p}$. Choose $x_{s}$ as in (2.3). If $x_{s} \in A_{2 q}^{p}$ its image in $Q\left(A^{p}\right)_{2 q}$ is nonzero and for dimensional reasons $q+(p-1)=0 \bmod m$.

The proof of Lemma 2.1 (b). Choose a basis for $P^{1}\left\{Q\left(A^{p}\right)_{2 q}\right\}$ and extend to a basis for the whole of $Q\left(A^{p}\right)_{2 m}$. We take representatives for this basis in $A_{2 m}^{p}$ as part of the set of generators of $A^{p}$. Suppose that $x_{1}$ is a generator whose image in $Q\left(A^{p}\right)_{2 m}$ does not lie in the image of $P^{1}$. Then clearly we cannot choose $x_{s}$ to satisfy (2.3). The contra- 
diction implies that $P^{1}: Q\left(A^{p}\right)_{2 q} \rightarrow Q\left(A^{p}\right)_{2 m}$ is surjective.

The proof of Lemma 2.1 (c). Suppose that $q \neq m-(p-1)$ or the result is trivial. Choose a basis for the kernel of $P^{1}: Q\left(A^{p}\right)_{2 q}$ $\rightarrow Q\left(A^{p}\right)_{2 q+2(p-1)}$ and extend to a basis of $Q\left(A^{p}\right)_{2 q}$. The images under $P^{1}$ of the additional vectors are linearly independent in $Q\left(A^{p}\right)_{2 q+2(p-1)}$ and we extend to a basis of $Q\left(A^{p}\right)_{2 q+2(p-1)}$. We take representatives in $A_{2 q}^{p}$ and $A_{2 q+2(p-1)}^{p}$ for both bases as part of the set of generators of the ring $A^{p}$. Now if $x_{1} \in A_{2 m}^{p}$ is a generator, again we cannot choose $x_{s}$ to satisfy (2.3). This completes the proof of Lemma 2.1.

The proof of Theorem 1.2. Suppose that $m \in t(X)$ and that the integers $r-1$ and $m$ are coprime. We choose a prime number $p$ of the form $k m-(r-1)$ which lies in $P$. This is possible as $k m-(r-1)$ is a prime number for an infinite number of integral $k$ [6]. Now $Q\left(A^{p}\right)_{2 m}$ $\neq 0$ as $m \in t(X)$ and since $m$ and $p$ are relatively prime, Lemma 2.1 (a) implies that $Q\left(A^{p}\right)_{2 q} \neq 0$ for some $q$ with $q+(p-1)=0 \bmod m$. But $r+(p-1)=0 \bmod m$ and so $q=r \bmod m$, where $q \in t(X)$. This completes the proof.

3. Let $\bar{X}$ be the universal covering complex of $X$. Then $\bar{X}$ is also an associative $H$-space which has the homotopy type of a finite $\mathrm{CW}$ complex [2]. Further $t(\bar{X})$ is just $t(X)$ in which each integer 1 has been omitted, again by the methods of [2]. Thus without loss of generality it is sufficient to prove Theorem 1.1 with the additional hypothesis that $X$ is simply connected. Using this additional hypothesis, we now apply Theorem 1.2 , when $r(X) \leqq 4$. First we consider its corollary. Let the integer $n$ have distinct prime power decomposition $p_{1}^{\alpha} p_{2}^{\beta} \cdots, \alpha>0, \beta>0, \cdots$, then it is well known and not difficult to prove that

$$
\phi(n)=p_{1}^{\alpha-1}\left(p_{1}-1\right) p_{2}^{\beta-1}\left(p_{2}-1\right) \cdots
$$

Thus if $m$ is the largest integer in $t(X)$, Corollary 1.3 implies that $m$ must be one of the numbers $2,3,2^{2}, 5,2 \cdot 3,2^{3}, 2 \cdot 5$, or $2^{2} \cdot 3$. We now consider the restriction put upon the possible choices for $t(X)$ which are implied by Theorem 1.2, where the largest integers are as described. Ignoring multiplicities, the possible sets are

$$
\begin{gathered}
\{2\},\{2,3\},\{2,4\},\{2,3,4\},\{2,3,4,5\},\{2,6\},\{2,3,6\},\{2,4,6\}, \\
\{2,3,4,6\},\{2,4,6,8\} \text { and }\{2,6,8,12\} .
\end{gathered}
$$

Now the examples for $t(G)$ which arise from Lie groups are unions of the following sets, where we take the examples in the order given after the statement of Theorem 1.1, excluding $S^{1}$, 


$$
\begin{gathered}
\{2\},\{2,3\},\{2,3,4\},\{2,3,4,5\},\{2,4\},\{2,4,6\},\{2,4,6,8\}, \\
\{2,4,4,6\},\{2,6\} \text { and }\{2,6,8,12\} .
\end{gathered}
$$

Thus to complete the proof of Theorem 1.1 we must eliminate all types listed below. The groupings are dictated by the particular argument which we shall use for the elimination.

$$
\begin{aligned}
& t(a):\{2,3,3\},\{2,3,3,3\} \text { and }\{2,3,3,4\}, \\
& t(b):\{2,4,4\},\{2,4,4,4\} \text { and }\{2,3,4,4\}, \\
& t(c):\{2,6,6\},\{2,6,6,6\},\{2,3,6,6\} \text { and }\{2,4,6,6\}, \\
& t(d):\{2,3,6\},\{2,3,3,6\} \text { and }\{2,3,4,6\} .
\end{aligned}
$$

To proceed further it is convenient to gain more information on the set of primes $P$. We deduce this information from three results of W. Browder.

(3.1) If $H^{*}(X, Z)$ has $p$-torsion, then $H^{*}\left(X, Z_{p}\right)$ is not an exterior algebra on odd dimensional generators.

(3.2) $\pi_{2}(X)=0$.

(3.3) If $H^{*}\left(X, Z_{p}\right)$ has a generator of dimension $2 n$, then $H^{*}(X, Q)$ has a generator of dimension $2 n p^{k}-1$ for some $k$ in $0<k<\infty$.

The first result is Theorem 4.9 of [3], the second is part of Theorem 6.11 of [3] and the third is part of Theorem 4.7 of [4].

Now suppose that $p \notin P$ where $p$ is an odd prime. Then by (3.1) the finite dimensional Hopf algebra $H^{*}\left(X, Z_{p}\right)$ has an even dimensional indecomposable element; see Theorem 6.1 of [1]. Thus by (3.3), $H^{*}(X, Q)$ has a generator of dimension $2 n p^{k}-1$ for some $k$ in $0<k<\infty$. In fact we can show that $n>1$, for the Hurewicz isomorphism theorem implies that $H_{2}(X, Z)=0$ since $\pi_{1}(X)=\pi_{2}(X)=0$, and so $H^{2}\left(X, Z_{p}\right)=0$.

If $t(X)$ is one of the sets listed under $t(a)$ or $t(b)$ this implies that $3 \in P$ since in all cases $H^{2 n 3^{k}-1}(X, Q)=0$ for $n>1$ and $k>0$. Similarly $5 \in P$ for the examples listed under $t(c)$ and $t(d)$, for all nonzero elements in $H^{2 n 5^{k}-1}(X, Q)$ are decomposable for $n>1$ and $k>0$. (An alternative argument replaces (3.3) by Theorem 7.1 of [3].)

We now consider $t(b), t(c)$ and $t(d)$. First we apply Lemma 2.1 (b) with $p=3$ and $m=4$ and deduce that for $t(X)$ as in $t(b), P^{1}: Q\left(A^{3}\right)_{4}$ $\rightarrow Q\left(A^{3}\right)_{8}$ is surjective. This is impossible for dimensional reasons. The argument to eliminate the examples of $t(c)$ is similar with $p=5$ and $m=6$. For $t(d)$ we first apply Lemma 2.1 (b) with $p=5$ and $m=6$ and deduce that $P^{1}: Q\left(A^{5}\right)_{4} \rightarrow Q\left(A^{5}\right)_{12}$ is an epimorphism and therefore an isomorphism. Lemma 2.1 (c) with $p=5$ and $m=3$ implies that $Q\left(A^{5}\right)_{6}=0$, which is false. It therefore remains to consider examples 
$t(a)$. Now arguments similar to those already used imply that if $t(X)=\{2,3,3\}$ or $\{2,3,3,3\}$, then $2 \in P$. Then applying Lemma 2.1 (b), or otherwise, these can be eliminated as in cases $t(b)$ and $t(c)$. However the author does not know how to prove directly that type $\{2,3,3,4\}$ has no 2-torsion in its integral homology. Therefore in the next section we give an argument eliminating all three examples using the prime $3 \in P$.

Finally it is natural to ask if the isomorphism of Theorem 1.1 established over the rationals also holds for the cohomology rings with other coefficients. Arguments as above imply that $p \in P$ for all primes $p \geqq 5$ and so here there is no problem. To establish an isomorphism with $Z_{3}$ coefficients by these methods, including type $\{2,6,8,12\}$, appears to need an additional assumption such as $\pi_{3}(X)$ being torsion free, but for the prime 2 even this additional hypothesis does not appear to lead to the result. The author hopes to return to such questions using different techniques at a later date.

4. We use the methods of [9] and [10] with $p=3$ and refer to these for the general theory. We provide the details needed to eliminate type $\{2,3,3,4\}$. The same argument works for $\{2,3,3\}$ and $\{2,3,3,3\}$ if the second paragraph of this section is omitted and $y_{4}$ is put equal to zero thereafter. The approach is analogous to that already used where $A^{3}$ is replaced by $B^{3}$, a truncated polynomial algebra of height 4 over $Q_{3}$, the ring of integers localized at the prime ideal (3), and $B^{3} \otimes Z_{3} \cong A^{3}$. The cyclic reduced powers are replaced by homomorphisms $S^{q}: B_{2 n}^{3} \rightarrow B_{2 n+4 q}^{3}$, where $S^{q}$ reduced modulo 3 is just $P^{q}$.

Lemma 2.1 (b) implies that $P^{1}: Q\left(A^{3}\right)_{4} \rightarrow Q\left(A^{3}\right)_{8}$ is surjective, since $4 \in t(X)$, and therefore for dimensional reasons it is an isomorphism. Let $y_{2}$ generate $B_{4}^{3}$ and set $y_{4}=S^{1} y_{2}$. Now $S^{2} y_{2}=y_{2}^{3} \bmod 3$, since $S^{2}$ $\bmod 3$ is just $P^{2}$, and so $S^{1} y_{4}=2 y_{2}^{3} \bmod 3$, since $P^{1} P^{1}=2 P^{2}$ by (2.2). We choose the basic splitting of the $K$-theory which defines the $S^{q}$ so that $S^{1} y_{4}=2 y_{2}^{3}$.

Now let $y_{3}, y_{3}^{\prime}, \cdots$ be representatives in $B_{6}^{3}$ for a basis of $Q\left(B^{3}\right)_{6}$. We decompose $B^{3}$ as $I \oplus J$, where $I$ is the ideal generated by $B_{4}^{3}$ and $B_{8}^{3}$, and $J$ is the subring with a multiplicative basis $y_{3}, y_{3}^{\prime}, \cdots$. Let $S^{2} y_{2}=z+3 q\left(y_{3}, y_{3}^{\prime}, \cdots\right)$ where $z \in I$ and $q$ is a quadratic expression in $J$. The homomorphism $Q^{q}: B_{2 n}^{3} \rightarrow B_{2 n+4 q}^{3}$ are related to the $S^{q}$ by $S^{0}=Q^{0}=$ Identity and $\sum_{i+j=n} S^{i} Q^{j}=0$ for each $n>0$. Let $Q^{1} y_{3}$ $=y_{2} l\left(y_{3}, y_{3}^{\prime}, \cdots\right)$ and $Q^{2} y_{3}=y_{2}^{2} m\left(y_{3}, y_{3}^{\prime}, \cdots\right)+y_{4} n\left(y_{3}, y_{3}^{\prime}, \cdots\right)$, where $l$ and $m$ and $n$ are linear expressions in their arguments. We now apply the relation 


$$
\left(1-k^{6}\right) S^{3}+k^{2}\left(1-k^{4}\right) S^{2} Q^{1}+k^{4}\left(1-k^{2}\right) S^{1} Q^{2}=0 \bmod 3^{3},
$$

where $k$ is not divisible by 3 ; see Corollary 2.10 of [9] of Corollary 2.11 of [10]. We consider the component in $J$ when (4.1) is applied to $y_{3}$. Now the homomorphisms $S^{q}$ can be defined so that they satisfy the usual Cartan formula, since $B^{3}$ is a truncated polynomial algebra. Hence $S^{1} Q^{2} y_{3}$ lies in $I$ and the component of $S^{2} Q^{1} y_{3}$ in $J$ equals $3 q\left(y_{3}, y_{3}^{\prime}, \cdots\right)$. If $k=2$, (4.1) becomes $9 S^{3} y_{3}+6 S^{2} Q^{1} y_{3}+3 S^{1} Q^{2} y_{3}=0$ mod 27, and since $S^{3} y_{3}=y_{3}^{3} \bmod 3$, we deduce that

$$
y_{3}^{3}=q\left(y_{3}, y_{3}^{\prime}, \cdots\right) l\left(y_{3}, y_{3}^{\prime}, \cdots\right) \bmod 3 \text {. }
$$

A similar argument applied to $y_{3}^{\prime}$ implies that

$$
{y_{3}^{\prime}}^{3}=q\left(y_{3}, y_{3}^{\prime}, \cdots\right) l^{\prime}\left(y_{3}, y_{3}^{\prime}, \cdots\right) \bmod 3,
$$

where $l^{\prime}$ is linear. These last two equations are clearly incompatible.

AdDEd IN Proof. After submitting this paper, the author discovered that the main results given here have also been known for some time to J. Ewing, a pupil of A. Clark.

\section{REFERENCES}

1. A. Borel, Sur la cohomologie des espaces fibrés principaux et des espaces homogènes de groupes de Lie compacts, Ann. of Math (2) 57 (1953), 115-207. MR 14, 490.

2. W. Browder, The cohomology of covering spaces of H-spaces, Bull. Amer. Math. Soc. 65 (1959), 140-141. MR $22 \# 1891$.

3. - Torsion in H-spaces, Ann. of Math. (2) 74 (1961), 24-51. MR 23 \#A2201.

4. - Differential Hopf algebras, Trans. Amer. Math. Soc. 107 (1963), 153-176. MR $26 \# 3061$.

5. A. Clark, On $\pi_{3}$ of finite dimensional H-spaces, Ann. of Math. (2) 78 (1963), 193-196. MR 27 \#1956.

6. P. Dirichlet, Werke. Band 1.XXI, pp. 313-342.

7. A. Dold and R. Lashof, Principal quasi-fibrations and fibre homotopy equivalence of bundles, Illinois J. Math. 3 (1959), 285-305. MR 21 \#331.

8. P. Hilton and J. Roitberg, On the classification problem for $\mathrm{H}$-spaces of rank 2, Comment Math. Helv. 45 (1970), 506-516.

9. H. Hopf, Ueber die Topologie der Gruppen-Mannigfaltigkeiten und ihrer Verallgemeinerungen, Ann. of Math. (2) 42 (1941), 22-52. MR 3, 61.

10. J. R. Hubbuck, Generalized cohomology operations and H-spaces of low rank, Trans. Amer. Math. Soc. 141 (1969), 335-360. MR 40 \#2059.

11. - Primitivity in torsion free cohomology Hopf algebras, Comment Math. Helv. 46 (1971), 13-43.

12. S. Ochiai, On the type of an associative H-space of rank three, Proc. Japan Acad. 44 (1968), 811-815. MR 39 \#6319.

13. - On the type of an associative H-space, Proc. Japan Acad. 45 (1969), 92-94. MR 40 \#3544. 
14. L. Smith, On the type of an associative $H$-space of rank two, Tôhoku Math. J. (2) 20 (1968), 511-515. MR 39 \#4844.

15. N. E. Steenrod and D. Epstein, Cohomology operations, Ann. of Math. Studies, no. 50, Princeton Univ. Press, Princeton, N. J., 1962. MR 26 \#3056.

16. A. Zabrodsky, Homotopy associativity and finite CW-complexes, Topology 9 (1970), 121-128.

17. A. Zabrodsky, The classification of simply connected H-spaces with three cells. I, II (to appear).

University of Manchester, Manchester, England

Gonville and Caius College, Cambridge, England 